Campdesuñer Sarquiz, L. \& Murillo Estepa, P. (2020). El conflicto en contextos culturalmente diversos desde la perspectiva de profesionales de la educación. Revista Electrónica Interuniversitaria de Formación del Profesorado, 23(3), 149-168.

DOI: https://doi.org/10.6018/reifop.388981

\title{
El conflicto en contextos culturalmente diversos desde la perspectiva de profesionales de la educación
}

\author{
Leila Campdesuñer Sarquiz ${ }^{1}$ Paulino Murillo Estepa² \\ ${ }^{(1)}$ Universidad Católica Silva Henríquez; ${ }^{(2)}$ Universidad de Sevilla
}

\section{Resumen}

Este artículo describe la visión de profesionales acerca del origen y naturaleza de los conflictos en interculturalidad en seis establecimientos educacionales municipalizados y culturalmente diversos de la comuna de Santiago, Chile, que se caracterizan por un aumento gradual de la matrícula de estudiantes inmigrantes. Los resultados expuestos son fruto de un estudio mayor de tipo mixto de profundización, respecto del alcance de sus planes de mejora para una convivencia democrática e intercultural. Para la primera fase cuantitativa, se diseña y aplica un cuestionario a 205 profesionales docentes y no docentes cuyos resultados fueron analizados a través del paquete estadístico SPSS. En la fase cualitativa, se seleccionaron de manera intencionada 26 profesionales quienes fueron entrevistados a partir de una guía semiestructurada, cuyos resultados se analizaron por medio del software Nvivo 10. Gracias a la triangulación, se concluye que en las visiones de los profesionales persiste la negación de la discriminación, enmascaramiento y/o ocultación de los conflictos y su visión como un problema para el afianzamiento de la vida democrática. A este fenómeno se le ha denominado "preámbulo en la autoconciencia del conflicto".

\section{Palabras clave}

Autoconciencia del conflicto; discriminación; cultura de la mediación; interculturalidad

\section{Contacto:}

Leila Campdesuñer Sarquiz, camp.leila@gmail.com. General Jofré 462, Santiago. Santiago de Chile. Código postal 8900000.

Proyecto de Investigación Doctoral "Convivencia democrática y plan de mejora: un estudio en escuelas interculturales de la comuna de Santiago, Santiago de Chile". 


\title{
The conflict in culturally diverse contexts from the perspective of professionals of education
}

\begin{abstract}
This article describes the education professionals' view about the origin and nature of conflicts in interculturality within six culturally diverse municipal educational settings of the municipality of Santiago, Chile, marked by the increasingly continuous incorporation of migrant students. The results shown are part of a mixed and large deepening study regarding the scope of improvement plans with a view to developing culturally diverse and democratic coexistence. As for the first quantitative phase, a questionnaire is designed and applied to 205 teachers and instructors whose results were analyzed through a statistical software SPSS. In the qualitative phase, 26 professionals of education were chosen and interviewed using a semi-structured interview, whose results were analyzed by means of software Nvivo 10. By implementing a triangulation strategy, it is concluded that the negation of discrimination, masking and / or conflict concealment remains in education professionals' view and this idea emerges as an issue for democratic life in interculturality. This phenomenon has been defined as "a preamble to selfconsciousness conflict"
\end{abstract}

\section{Keywords}

Conflict self-awareness; discrimination; culture of mediation; interculturality.

\section{Introducción}

El presente artículo es fruto de una investigación que ha indagado acerca de las visiones de profesionales de establecimientos educacionales municipalizados sobre aspectos procedimentales y temáticos que subyacen en la fundamentación de planes de mejora en convivencia democrática. A raíz de la importancia del trabajo de prevención de la discriminación en diversidad cultural, enmarcado en una política educativa orientada hacia la inclusión, guarda especial relevancia conocer las visiones de los profesionales de los centros respecto al origen y la naturaleza de los conflictos en contextos culturalmente diversos, ya que las mismas están en la base del diseño de las mejoras de la vida democrática de las escuelas.

Si bien en Chile el fenómeno migratorio es de larga data, subsiste una historia de discriminación hacia algunos grupos de inmigrantes, por no coincidir con la "aspiración a la blancura" (Correa, 2016) que está presente en la configuración de la identidad cultural nacional.

Estas manifestaciones de discriminación —que devienen en barreras para la integración de estudiantes con características raciales que se contraponen al imaginario identitario de un chileno "europeo, blanco" (Tijoux, 2016) — se reproducen en el ámbito escolar. Estudios llevados a cabo en contextos escolares chilenos, como los de Riedemann y Stefoni (2015) y Tijoux (2013a; 2013b) dan cuenta de un escenario complejo para los estudiantes inmigrantes, particularmente 
peruanos y haitianos: "La discriminación que cotidianamente viven los hijos de inmigrantes peruanos en Chile es producto de la condición de origen de sus padres, y que el racismo contenido en esa discriminación obstaculiza su inserción en el país” (Tijoux, 2013a, p. 102).

Por su parte, Stefoni (2010) ya ha constatado que los escolares peruanos enfrentan maltratos físicos y ofensas, lo que más recientemente también está ocurriendo con los estudiantes haitianos (Riedemann y Stefoni, 2015), reproduciendo así etiquetas raciales que avergüenzan de su propia singularidad al discriminado. Sin embargo, llama la atención la permanente negación de la discriminación por parte de integrantes de comunidades educativas, la que ha sido constatada por estos autores en sus investigaciones, quienes proponen tipologías de intencionalidad racista en el discurso de profesionales: negación directa; negación de intencionalidad racista (a través del uso de bromas); mitigación (minimización con el uso de eufemismos y justificaciones) y acusación de racismo revertida (referencias a exageraciones o ver discriminación donde no existe) (Riedemann y Stefoni, 2015).

Estos hallazgos dan cuenta de que en las comunidades educativas persisten manifestaciones de exclusión a partir de prejuicios instalados en el imaginario social, lo cual va de la mano con la visión que se tiene acerca del conflicto en diversidad cultural, tema que ha devenido como un factor psicosocial predisponente de discriminación.

El conflicto en interculturalidad se produce a partir de una variedad de significados, desacuerdos, expectativas incumplidas e insatisfacciones relacionadas con factores culturales y emocionales, por lo cual debe ser visto como un fenómeno inevitable y cotidiano, pero necesario para una convivencia democrática, participativa, desde el respeto a la singularidad, que va más allá de la tolerancia para la coexistencia desde la multiculturalidad. Es así que el conflicto en interculturalidad se expresa como:

aquel que hace referencia a toda situación escolar donde acontezca una divergencia entre miembros de diferentes grupos culturales que convivan en un centro educativo por cuestiones de índole cultural (incomunicación entre familias y entre alumnos de diferentes culturas, malentendidos por empleo de códigos de referencia cultural distinta) o también a una situación de desequilibrio derivado por una compleja red de significados (afectivos, políticos, éticos), definidos de forma implícita u oculta (Leiva, 2012, p. 60).

El conflicto en interculturalidad deviene como un espacio sociopsicológico para el desarrollo de la convivencia democrática, la interdependencia, el reconocimiento de singularidades, la cooperación y la formación de valores como el respeto y la solidaridad, todo lo cual mantiene a raya expresiones de discriminación y exclusión en los entornos escolares.

\section{Antecedentes más relevantes del estudio}

El concepto de interculturalidad es complejo, describe una relación entre culturas e implica interacción (Marín y Venegas, 2009). Dado lo anterior, la educación intercultural se dirige a concientizar para la aceptación de la diversidad e interdependencia (Essomba, 2006) a partir del respeto, la valoración de la diversidad cultural y la superación de la discriminación (Arnaiz, 2012). 
Lo anterior ha suscitado la proliferación de estudios acerca del conflicto intercultural a lo largo de las últimas décadas. Se comparte con Leiva (2012, 2015) que en sus inicios, estas investigaciones fueron más del tipo cuantitativo y no permitieron una comprensión profunda del tema. Sin embargo, en los últimos años la investigación cualitativa y los estudios mixtos de profundización han demostrado ser una vía para ahondar en significados asociados, como es el caso de diversos autores europeos (Díaz, 2006; Jordán y Castella, 2001; Leiva, 2010; 2012; 2015; 2016; 2017; Soriano, 2004).

Por su parte, en América Latina han emergido estudios de aproximación teórica sobre educación intercultural de carácter multidisciplinario, de evaluación educativa, sociocultural, sociolingüístico y etnográfica, que privilegian la migración y diversidad étnica en el sistema formal. De acuerdo con Villalta (2016) algunos de estos estudios ponen el acento en la migración, la diversidad étnica y la innovación en educación intercultural desde bases identitarias (BarrigaVillanueva, 2008; Castro, 2011; Cebrián y Noguera, 2010; Dumrauf y Menegaz, 2013;).

Desde una mirada crítica, guardan especial relevancia los trabajos de Walsh (2009) sobre interculturalidad crítica, aula intercultural y las prácticas de re-existencia. Por otro lado, son igualmente significativos los estudios de Mignolo $(2007,2008)$ y sus unidades de análisis para la investigación, tales como "diferencia colonial", "pensamiento fronterizo", "colonialidad del ser".

Durante los años 80 y 90 del siglo XX, la diversidad cultural es tema central en las reformas educacionales de Latinoamérica por medio de Programas de Educación Bilingûe, cuya intencionalidad no ha sido repensar los sistemas educativos, sino acomodar un discurso de la diversidad e interculturalidad desde una convivencia tolerante y de reconocimiento de la diferencia cultural, sin mayor repercusión (Walsh, 2009).

En el caso de Chile, actualmente el Ministerio de Educación impulsa este programa a través del currículo nacional, ante la pérdida de la lengua y cultura de los pueblos originarios, y sobre todo para alcanzar la paz, la igualdad, la prevención de la violencia y la discriminación en todas sus formas.

Durante los últimos años el país ha mostrado un incremento significativo de familias inmigrantes, sus establecimientos escolares están afrontando el desafío de la prevención de la discriminación. Ante ello han emergido nuevos estudios que generan bases psicoeducativas para promover una educación sin condicionamientos arbitrarios, entre ellos, la cultura y etnia.

Sus propuestas se orientan desde la riqueza de la interculturalidad, que supera la visión multicultural de coexistencia, paz y tolerancia. La prevención de la discriminación en el contexto escolar supone la interacción de diversos grupos culturales desde el respeto a la singularidad por sobre la tolerancia, y para ello es necesario el desarrollo del auto-concepto en las minorías étnicas (Bank, 2008), tema de inicio para una interacción que se sustente en el respeto a la singularidad cultural.

En el caso puntual de los resultados que se presentan sobre las percepciones de profesionales acerca del conflicto intercultural, entre otros antecedentes se consideran los relativos al rol de los profesionales en la escuela intercultural. Aguado (2008) reconoce que pese al discurso de un profesorado que avala la diversidad como algo enriquecedor, en la práctica evidencia una 
percepción problemática de la misma que se conecta con una serie de demandas, entre las cuales se encuentra "¿cómo prevenir los conflictos xenófobos en los centros educativos?" o "¿cómo integrar su cultura en la escuela?" (Aguado, 2008, p.276)

Los estudios de Leiva (2010; 2012; 2013; 2015; 2016; 2017) en Málaga, Andalucía, sobre las perspectivas del profesorado acerca del conflicto en escuelas interculturales por igual evidencian una complejidad de significados acerca del tema, la necesidad de lograr la participación de las familias inmigrantes y autóctonas en la vida escolar, así como de una cultura de la mediación.

Los hallazgos del autor ofrecen pistas para la mejora de la convivencia democrática en escuelas interculturales de Chile. Por un lado, el conocimiento de las percepciones de sus profesionales sobre el conflicto intercultural como espacio sociopsicológico para educar en valores y prevenir la discriminación. Por otra parte, la importancia de desarrollar competencias interculturales en los profesionales e incentivar una cultura de la mediación en interculturalidad hacia el interior de los centros educativos, así como involucrar la reflexión constante de la comunidad educativa y la mejora de la colaboración familias-escuela.

Estos antecedentes, así como los hallazgos de Riedemann y Stefoni (2015) sobre la discriminación en escenarios escolares chilenos, han sido relevantes en la indagación de las perspectivas sobre el origen y naturaleza del conflicto en interculturalidad por parte de profesionales de seis establecimientos educacionales culturalmente diversos de la comuna de Santiago, Chile.

\section{Comprensión acerca del origen y naturaleza del conflicto en interculturalidad}

De acuerdo con Bartolomé (2002) la escuela intercultural nace del conflicto como expresión que conjuga características personales y sociofamiliares, así como la estructura de la organización y la cultura escolar. Por lo tanto, el conflicto en interculturalidad es multicausal y necesario como espacio de intercambio de significados e influencia recíproca, modulado por los procesos emocionales, donde el individuo se reconoce en la interacción como sujeto cultural activo.

Con ello, la educación desde el conflicto es algo positivo y necesario para el desarrollo personal y la convivencia en diversidad cultural, la participación y el respeto, como una vía para la prevención de la discriminación. Sin embargo, esto contradice la idea comúnmente arraigada de que la ausencia de conflictos entre el alumnado es sinónimo de buena convivencia (IbarrolaGarcía e Iriarte, 2013), como es el caso del contexto educacional chileno, donde esta creencia representa una de las razones por las cuales no se evidencia una clara tendencia hacia la fundamentación de propuestas que sigan la línea de la educación intercultural entendida como un proceso dinámico orientado a la aceptación de la diversidad cultural, la interdependencia y evolución hacia un nuevo y más enriquecedor concepto de sociedad y ciudadanía (Essomba, 2006).

Es por ello por lo que conocer las visiones de profesionales de la educación acerca del conflicto permite develar las barreras que impiden la prevención de la discriminación, las causas que subyacen a la ausencia de un trabajo encaminado al desarrollo de una cultura de la mediación y 
la formación de valores desde el espacio mismo del conflicto, así como de un debate reflexivo en torno a lo que hoy es la escuela en el marco del cambio paradigmático.

A partir de los antecedentes mencionados, desde el punto de vista metodológico se considera que el origen y naturaleza de los conflictos en interculturalidad se configura a partir del vínculo de dos unidades de análisis, tal como se aprecia en la Figura 1:

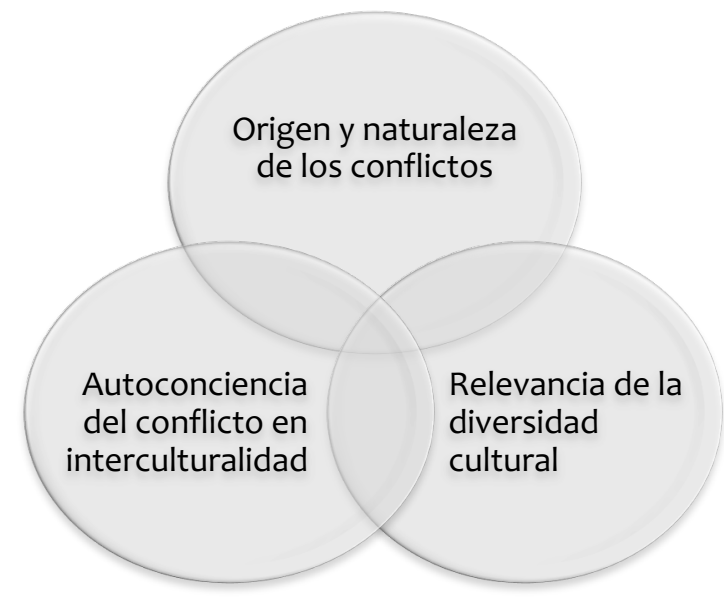

Figura 1. Configuración del origen y naturaleza de los conflictos en interculturalidad Fuente: Elaboración propia.

Al profundizar en la unidad de análisis "autoconciencia del conflicto en interculturalidad" como condición inicial para su afrontamiento, las teorías implícitas de los profesionales se pueden agrupar en el siguiente continuum:

- Visibilidad del conflicto intercultural y de su carácter inevitable. Evidencia la sensibilización con el mismo, su reconocimiento para potenciar una cultura de la participación, respeto a la singularidad cultural y la vida democrática.

- Ocultación y/o no reconocimiento del conflicto en interculturalidad. Evidencia la negación del conflicto y su "demonización”, que es una barrera para el afianzamiento de la vida democrática y prevención de la discriminación.

- Enmascaramiento del conflicto. Expresa la resistencia pasiva al reconocimiento del conflicto intercultural.

Por su parte, las visiones respecto de la "relevancia de la diversidad cultural" pueden agruparse de la siguiente manera:

- La diferencia cultural es relevante para la aparición de conflictos. Significa que la singularidad cultural puede dar lugar a desencuentros valóricos, éticos, identitarios y de comunicación, entre otros. Tiene valor positivo para los procesos de integración y reconfiguración de la identidad personal y cultural de estudiantes inmigrantes y autóctonos, así como de los profesionales de los centros, que se ven enriquecidos en este concierto de diversidad. 
- $\quad$ La diferencia cultural es irrelevante para la aparición de conflictos. Significa desconocer la importancia de la singularidad cultural y del conflicto intercultural como condición inevitable y necesaria para la prevención de la discriminación.

- $\quad$ Enmascaramiento de la diferencia cultural. Resistencia pasiva a lo diverso como riqueza y condición natural necesaria para la vida democrática en interculturalidad.

\section{Metodología}

\section{Diseño}

Se trata de una investigación educativa desarrollada en el año 2018 en contextos educativos culturalmente diversos. Es un estudio descriptivo, de tipo mixto de profundización, con un diseño de dos fases que combinó una primera etapa cuantitativa y una segunda cualitativa, conectadas lógicamente. Esto ofreció complejidad y profundidad a los hallazgos a partir de su vinculación y contrastación, por medio de la triangulación de datos.

Una de las interrogantes de la investigación en la que se enmarcan los resultados que se presentan ha sido ¿cuál es la visión de la comunidad educativa acerca de los aspectos procedimentales y temáticos que subyacen en el diseño e implementación de los planes de mejora en convivencia democrática en los centros educativos?

Dentro de estos aspectos temáticos, están las visiones de profesionales acerca del origen y naturaleza de los conflictos en contextos culturalmente diversos, sobre la interculturalidad, los enfoques para afrontar la diversidad y prevenir la discriminación, así como sus percepciones sobre la existencia de prejuicios, igualitarismo y de las disposiciones pedagógicas que subyacen al diseño de acciones educativas para afrontar la diversidad (Leiva, 2012, 2016) a través del plan de mejora.

Uno de los aspectos temáticos de mayor relevancia para la prevención de la discriminación y para el trazado de una estrategia formativa de los profesionales de los centros, es el referido al origen y naturaleza de los conflictos en interculturalidad. Por lo tanto, ha sido un objetivo conocer las visiones de profesionales de los centros estudiados acerca del tema, si las mismas resultaban ser un facilitador, o bien, un obstáculo para la fundamentación de planes de mejora en convivencia democrática y prevención de la discriminación.

\section{Población y Muestra}

Dadas las características del estudio, de tipo mixto de profundización con un diseño de dos fases y secuencial, se ha contado con una muestra cuantitativa y otra cualitativa. El universo consideró el conjunto de establecimientos educacionales municipalizados de la comuna de Santiago, Chile, caracterizados por el incremento progresivo de la matrícula estudiantes inmigrantes. Puesto que la muestra es un subconjunto representativo de los elementos pertenecientes a la población, a continuación se describen los criterios de selección de ambas muestras. 


\section{Muestra cuantitativa}

Para la selección de la muestra cuantitativa de la primera fase del estudio, se tomaron en cuenta las características de los centros educativos y de los participantes. En cuanto a los centros, se seleccionaron de manera intencionada seis establecimientos educacionales que, en su conjunto, aportaron 205 profesionales de la educación formal pública, bajo un criterio de muestreo no probabilístico intencionado. Según un criterio estratégico o teórico de muestreo se tomaron en cuenta el nivel de enseñanza a petición de la Dirección de Educación de la Municipalidad de Santiago, que hizo explícito su interés por las escuelas de enseñanza básica.

Por otro lado, se tomó en cuenta el porcentaje de estudiantes inmigrantes matriculados, de acuerdo con el seguimiento realizado por la Subdirección de Desarrollo Social de dicha Municipalidad. Por último, se consideró la disposición de los centros a participar en el estudio. La Tabla 1 permite visualizar la muestra cuantitativa.

Tabla 1.

Datos de los centros educativos seleccionados en la muestra cuantitativa

\begin{tabular}{lccc}
\hline Centros & $\begin{array}{c}\text { Estudiantes } \\
\text { inmigrantes }\end{array}$ & Matrícula total & $\begin{array}{c}\text { \% Estudiantes } \\
\text { inmigrantes }\end{array}$ \\
\hline 1 & 168 & 363 & $46,3 \%$ \\
2 & 88 & 589 & $14,9 \%$ \\
3 & 40 & 639 & $18,5 \%$ \\
4 & 88 & 597 & $14,7 \%$ \\
5 & 324 & 626 & $51,8 \%$ \\
6 & 61 & 209 & $29,2 \%$ \\
\hline
\end{tabular}

Fuente: Elaboración propia.

Para la selección de los participantes, de los 205 involucrados, de manera intencionada se incorporaron profesionales docentes y no docentes, integrantes de equipos directivos y de mejora de los establecimientos educacionales. Esto obedeció, en primer lugar, a que todos los profesionales son dinamizadores y protagonistas en la construcción de una escuela inclusiva y democrática. En segundo término, a que el trabajo orientado al afianzamiento de la vida democrática y la prevención de la discriminación guarda relación con las perspectivas de todos los profesionales, por configurar teorías implícitas que marcan modos de relacionarse y construir estrategias orientadas al trabajo en interculturalidad. De acuerdo con ello, la distribución de los 205 casos queda reflejada en la Tabla 2:

\section{Muestra cualitativa}

La selección se verificó por juicio o intencionadamente por medio de la asignación de criterios heterogéneos de interés a lo largo de la primera fase cuantitativa, como el tipo de escuela y la concentración de estudiantes inmigrantes, el cargo y el rol que cumplen los profesionales. A partir de lo anterior, se configuró una muestra representativa y heterogénea constituida por 26 
participantes de los seis centros, que permitió profundizar en las perspectivas acerca del origen y naturaleza de los conflictos a partir de los hallazgos de la primera fase cuantitativa.

Tabla 2.

Datos de participantes de la muestra cuantitativa

\begin{tabular}{lcc}
\hline & $\mathrm{Fr}$ & $\%$ \\
\hline Equipo directivo/mejora & 20 & 9,8 \\
$\begin{array}{l}\text { Profesores segundo, } \\
\text { tercero y cuarto básico }\end{array}$ & 155 & 75,6 \\
No docentes & 30 & 14,6 \\
Total & 205 & 100,0 \\
\hline
\end{tabular}

Fuente: Elaboración propia.

La Tabla 3 muestra la configuración de la muestra cualitativa.

Tabla 3.

Datos de participantes de la muestra cualitativa

\begin{tabular}{ll}
\hline Criterios & Indicador \\
\hline Tipo de institución & Municipal \\
& \\
& \\
\hline Estudiantel de enseñanza: educación básica \\
& Concentración de estudiantes inmigrantes \\
\hline Equipos y profesionales & Cargo: Sí/No \\
& Equipo de mejora \\
& Equipo directivo \\
& Docente \\
& No docente
\end{tabular}

Fuente: Elaboración propia.

\section{Instrumentos}

De acuerdo con el enfoque mixto en el que se enmarca el estudio, se utilizó el cuestionario para la primera fase cuantitativa, y la entrevista semiestructurada para la segunda fase cualitativa. 
En el año 2018, para la primera fase, la investigadora diseñó, validó y aplicó el cuestionario para la evaluación de planes de mejora en convivencia democrática "Convivencia democrática en escuelas interculturales" (CDEI) del tipo escala valorativa, constituido por cinco categorías de respuestas: Muy de acuerdo (MA); de acuerdo (A); ni en acuerdo ni en desacuerdo (NA_ND); en desacuerdo (D); muy en desacuerdo (MD).

Por medio del cuestionario, fue posible conocer las visiones de profesionales de los centros estudiados acerca de los aspectos temáticos, entre ellos, el origen y naturaleza de los conflictos en interculturalidad, si es facilitador, o bien, obstáculo para la fundamentación de los planes de mejora en convivencia democrática y prevención de la discriminación.

El cuestionario ofrece 52 ítems agrupados en dos grandes dimensiones: procedimental y temática, y para determinar su valor y consistencia, se utilizó el criterio de expertos así como la medida de consistencia interna alfa de Cronbach, que alcanzó un 0,922.

Por otro lado, se llevó a cabo una prueba de análisis factorial que permitió comprender y resumir el comportamiento del origen y naturaleza de los conflictos, junto al resto de las categorías involucradas.

Para llegar a su versión final, a inicios del 2018 se realizaron dos pruebas piloto en dos establecimientos educacionales de la comuna de Recoleta, Santiago de Chile, con similares características a los centros que participaron en el estudio, lo que permitió contar con un instrumento acorde con las características culturales y del contexto educacional chileno y, de ese modo, acceder a las perspectivas de los profesionales respecto del origen y naturaleza de los conflictos en interculturalidad. Esta fase tuvo una duración de 6 meses.

Para la segunda fase cualitativa que tuvo una duración de 4 meses, se diseñó y validó una guía de entrevista semiestructurada dirigida a 26 profesionales, que dado su carácter flexible y abierto, resultó factible para profundizar y contrastar información a lo largo de ambas fases del estudio. Para su diseño se tomaron en cuenta los resultados de la primera fase cuantitativa, y para determinar su validez se utilizó el juicio de expertos, en este casos 17 académicos doctorados en el área de las Ciencias Sociales y de la Educación, formadores e investigadores con experiencia y publicaciones en temas afines al del estudio que se presenta.

Para garantizar el resguardo de la calidad y el rigor metodológico se utilizaron los criterios de dependencia, credibilidad y confiabilidad. En el caso de la dependencia, se recurrió a la validez interna. En cuanto a la credibilidad, se verificó que los entrevistados aportaran lo que considerasen adecuado reflexionar y expresar, no lo que realmente vivenciaban en la cotidianeidad. Para ello fue de gran valor el registro de citas textuales plasmadas en las transcripciones como respaldo, así como disponer de un cúmulo de información emergida en la primera fase del estudio. Por último, la confiabilidad fue posible al obtener información de diferentes fuentes: profesionales docentes, no docentes, directivos e integrantes de equipos de mejora.

A partir de este resguardo metodológico y de las sugerencias de los expertos, se contó con una guía de entrevista semiestructurada de 24 preguntas dirigida a profundizar en las perspectivas de los profesionales acerca de los aspectos temáticos, entre ellos, el origen y naturaleza del 
conflicto en interculturalidad: ¿se producen conflictos o roces entre estudiantes de distinto origen o país? ¿Qué tipos de conflictos? ¿Puede mencionar algunos ejemplos? ¿Por qué usted cree que ocurre esto? ¿Qué acciones llevan a cabo los equipos de gestión y mejora del establecimiento ante esos sucesos? ¿Qué criterios les merece el impacto de estas acciones? ¿Qué haría usted? Durante su aplicación se veló por la garantía ética y de confidencialidad de los 26 profesionales entrevistados.

\section{Procedimiento de recogida y análisis de los datos}

El análisis cuantitativo de la información recopilada por medio del cuestionario CDEI se realizó a través del programa SPSS versión 25.0. Se elaboraron tablas de frecuencia y se combinaron con sus respectivos gráficos, para conocer las perspectivas sobre los aspectos procedimentales y temáticos.

Los procedimentales se orientaron, principalmente, a conocer las percepciones sobre las dificultades y fortalezas existentes hacia el interior de los centros para el diseño e implementación de planes de mejora en convivencia democrática en contextos culturalmente diversos, sus visiones acerca de la interculturalidad, de las asesorías o capacitaciones necesarias para a un mayor empoderamiento en esta materia, los recursos materiales con que cuentan sus establecimientos, tiempos disponibles, entre otros.

Respecto a los temáticos, los ítems abordaron las percepciones sobre la diversidad cultural, la interculturalidad y las actitudes hacia la misma, la existencia de prejuicios, disposiciones pedagógicas para el diseño de acciones educativas con vista a la mejora de la convivencia democrática en interculturalidad, el conflicto intercultural y los valores para la convivencia en diversidad cultural. En particular, los ítems relacionados con el origen y naturaleza de los conflictos, fueron los siguientes:

26. La presencia cada vez mayor de estudiantes inmigrantes en su establecimiento ha aumentado los problemas de convivencia.

27. Los problemas de convivencia en su establecimiento tienen su origen en las diferencias culturales de los estudiantes.

Para llevar a cabo el análisis de la distribución de frecuencias y la interpretación de las perspectivas de los profesionales en torno al conflicto en interculturalidad como facilitador $u$ obstáculo en la prevención de la discriminación, se agruparon los datos en tres polos:

- $\quad$ Polo positivo: se agrupan las respuestas Muy de acuerdo y De acuerdo. Expresa una fortaleza.

- $\quad$ Polo negativo: se agrupan las respuestas Desacuerdo y Muy en Desacuerdo. Expresa una debilidad.

- $\quad$ Polo neutral: se agrupan las respuestas Ni de acuerdo ni en Desacuerdo. Expresa una amenaza.

Por su parte, para el análisis de los datos cualitativos y con vista a asegurar la confidencialidad e identificación de los participantes en la transcripción de las entrevistas se generaron códigos 
para cada uno de ellos, de modo que se contara con los datos relevantes para la posterior interpretación y discusión, tales como el número de la entrevista, el centro educativo y el tipo de estamento.

En el análisis e interpretación de los datos se utilizó el software Nvivo 10, que permite hallar perspectivas en datos no estructurados. La información fue organizada de manera que se pudieran detectar aspectos contundentes en las perspectivas de los profesionales, a manera de nodos y nubes de palabras. Esto facilitó la interpretación y contrastación de los datos, sus vínculos internos, y otorgar un sentido a las afirmaciones.

Finalmente, se llevó a cabo la triangulación de la información recopilada en ambas fases del estudio con vista a las discusiones y conclusiones finales.

\section{Resultados}

Para la interpretación de las unidades de análisis que configuran el origen y naturaleza de los conflictos en interculturalidad, respecto de la "autoconciencia del conflicto en interculturalidad" se consideró:

- Visibilidad del conflicto intercultural y su carácter inevitable. Muy de acuerdo y De acuerdo. Se expresa como fortaleza.

- $\quad$ Ocultación y/o no reconocimiento del conflicto en interculturalidad. Muy en Desacuerdo y En Desacuerdo. Se expresa como debilidad.

- $\quad$ Enmascaramiento del conflicto. Ni de acuerdo ni En Desacuerdo. Se expresa como amenaza.

Respecto a la "relevancia de la diversidad cultural" en el origen de los conflictos, se consideró:

- La diferencia cultural es relevante para la aparición de conflictos en la convivencia. Muy de Acuerdo y De Acuerdo. Es una fortaleza el reconocimiento de la singularidad cultural para la manifestación del conflicto como un espacio de educación en diversidad cultural.

- La diferencia cultural es irrelevante en la aparición de conflictos en la convivencia. Muy en Desacuerdo y En Desacuerdo. Es una debilidad desconocer la relevancia de la singularidad cultural y el conflicto en interculturalidad.

- $\quad$ Enmascaramiento de la diferencia cultural. Ni de acuerdo ni En Desacuerdo. Es una amenaza la resistencia pasiva a la riqueza de la singularidad en interculturalidad.

A continuación, en la Figura 2 se observan los hallazgos transversales en el comportamiento de las visiones de los profesionales participantes en el estudio. 


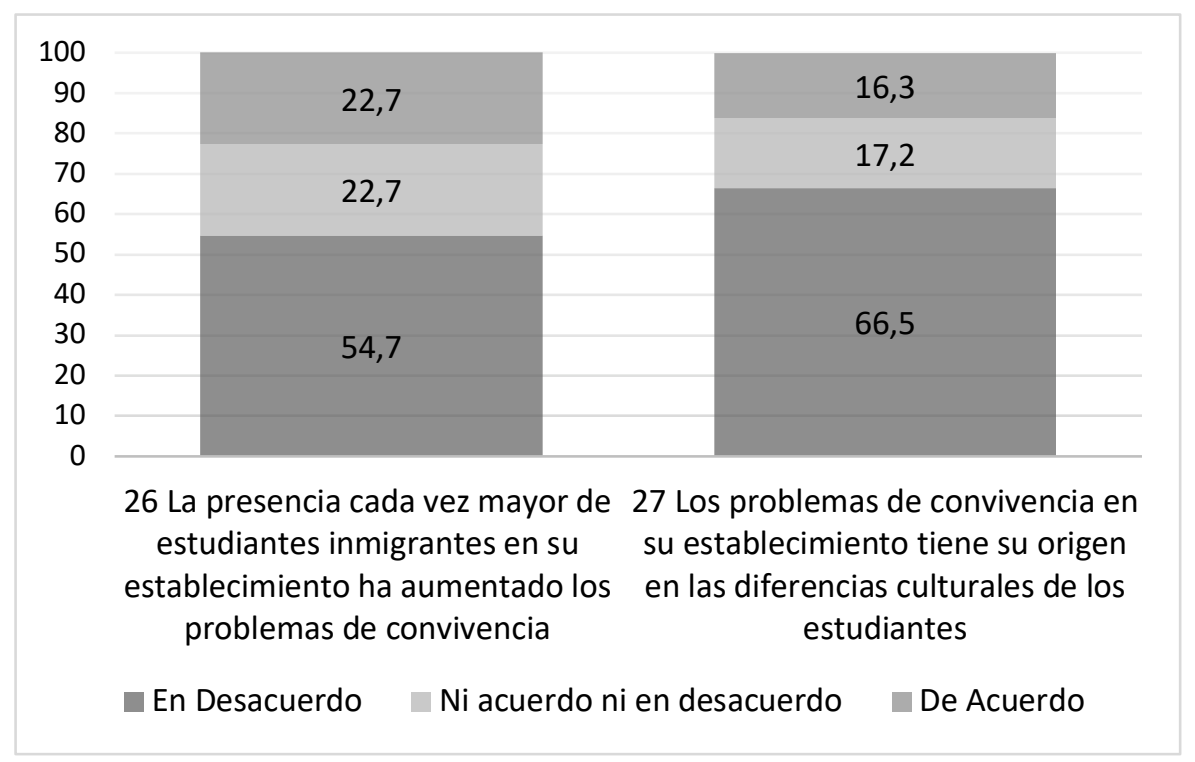

Figura 2. Visiones transversales del origen y naturaleza de los conflictos en interculturalidad Fuente: Elaboración propia.

Como se observa, el hecho de que un significativo 54,7\% de profesionales evidencie ocultación y/o no reconocimiento del conflicto en interculturalidad, es una debilidad para el diseño de acciones educativas encaminadas al trabajo desde la singularidad y una barrera para la prevención de la discriminación y el afianzamiento de la vida democrática hacia el interior de los centros. Estos resultados son coherentes con el $66,5 \%$ de profesionales que considera que la diferencia cultural es irrelevante en la aparición de conflictos en la convivencia en interculturalidad.

Al profundizar en los anteriores resultados a través del análisis de las entrevistas, se evidencia una diversidad de contenidos en las teorías implícitas de los profesionales, tanto para la visibilidad del conflicto como en la relevancia que le ofrecen a la diferencia cultural en su origen.

Ante algunas interrogantes, como aquellas dirigidas a conocer si se producen roces entre estudiantes de los distintos países y por qué, se pudo constatar la existencia de lo que se ha denominado "preámbulo en la autoconciencia del conflicto intercultural”.

Estos hallazgos significan que en torno a la autoconciencia de la existencia de conflictos en interculturalidad, emerge una variedad de teorías implícitas a partir de las configuraciones que adoptan la visibilidad del conflicto y el reconocimiento de la diversidad cultural. A continuación, la Figura 3 resume el fenómeno descrito. 


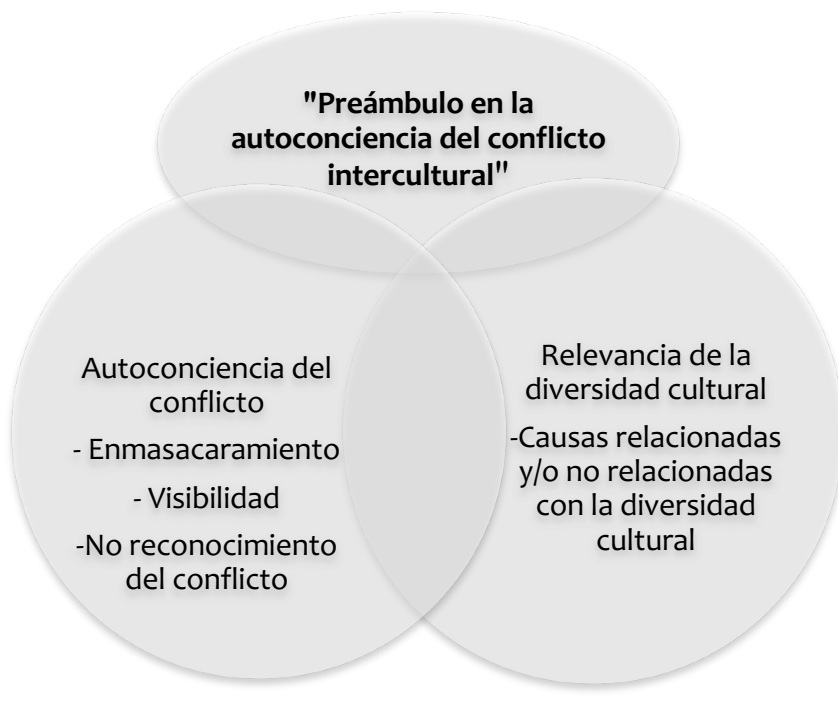

Figura 3. Preámbulo en la autoconciencia del conflicto Fuente: Elaboración propia.

Al analizar las visiones de los profesionales acerca de la "relevancia de la diversidad cultural" en la existencia de conflictos en interculturalidad, la Figura 4 permite visualizar lo polifacéticas que estas resultan no solo respecto de su importancia en la aparición de los conflictos, sino también en la autoconciencia y visibilidad de los conflictos en interculturalidad.

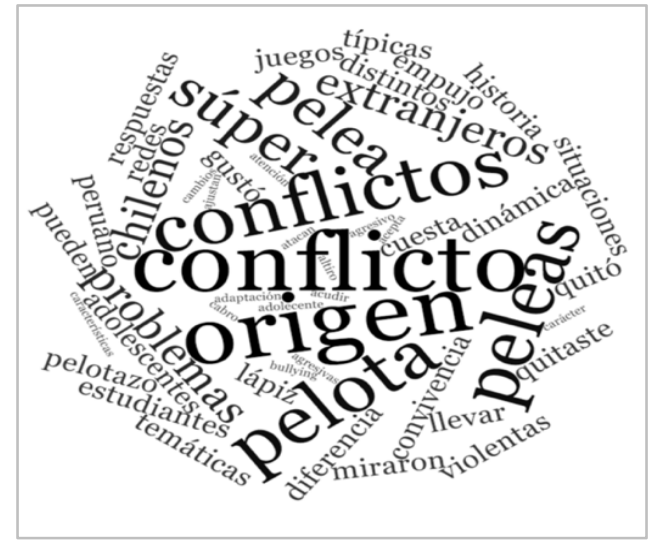

Figura 4. Nube: visibilidad del conflicto, causas relacionadas con la diversidad cultural.

Fuente: Elaboración propia.

De acuerdo con estos hallazgos, llama la atención la variedad de perspectivas en los profesionales, las que abarcan desde aquellas que consideran la posibilidad del conflicto, pero no deliberadamente vinculado con la diversidad cultural: "puede haber conflictos a veces, pero 
por un tema de que no se entienden en el uso de las palabras que son tan distintas de un lugar a otro" (Profesional, Centro 2). Por otro lado, se reconoce la existencia de conflictos y la relevancia de las diferencias culturales: "sí, se producen. Yo siento que es por la cultura de algunos, que son más confrontacionales y producen chismes, lo que no deja la tranquilidad que uno quisiera" (Profesional Centro 2). Así también, aparecen perspectivas que muestran ausencia de autoconciencia: "en realidad no hay conflictos que tengan que ver con la cultura en específico. Los que ocurren es porque un niño le dio un pelotazo a otro, más que todo" (Profesional Centro 4).

Por otra parte, se ha evidenciado una clara ocultación y/o no reconocimiento del conflicto en interculturalidad y de la relevancia de la diferencia cultura: "Los extranjeros que llegan acá se dan cuenta de que son tan bien bienvenidos que no se producen roces entre ellos y los estudiantes chilenos" (Profesional Centro 6). "Es difícil verlo, porque los niños se llevan generalmente bien. De existir, sería por pequeñeces, pero no por cosas así graves" (Profesional (entro 5).

En cuanto al enmascaramiento del conflicto, este se caracteriza por el reconocimiento de roces entre los escolares, pero minimizan sus causas y efectos, lo que expresa una resistencia pasiva a la singularidad y su riqueza cultural y, a la vez, como condición natural en los contextos educacionales. De este modo, si se manifestaran, ello no significaría que los niños tienen la intención de discriminar, sino que ello obedecería a conductas normales en esta etapa de desarrollo. La Figura 5 visualiza estos hallazgos.

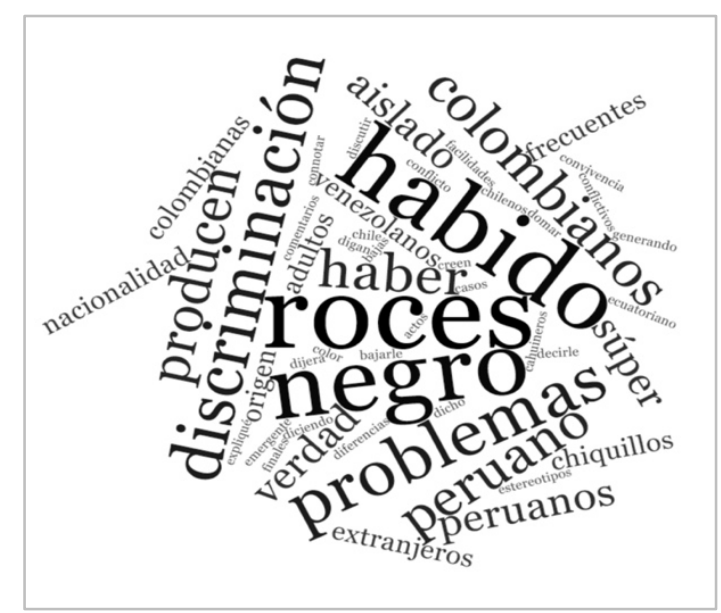

Figura 5. Nube: enmascaramiento del conflicto en interculturalidad.

Fuente: Elaboración propia.

A aquellas visiones que minimizan sus causas y efectos, se les ha denominado "enmascaramiento por atenuación": "No podemos decir que no se producen, porque en cualquier grupo de personas hay roces, y eso no significa que sea algo malo y que no se reconozcan" (Profesional 
Centro 3). "Trabajamos mucho con los estudiantes, cómo piensan y lo que sienten, y yo te diría que contadas veces se quejan de que me dijiste 'negro', o 'peruano' tal por cual” (Profesional Centro 2).

Junto con el "enmascaramiento por atenuación", también se ha evidenciado el "enmascaramiento por naturalización y/o transversalización": "De repente algunos chicos se refieren a otros, como cuando dicen "negro, ven", pero entonces estos me reconocen que no lo han dicho por algo malo, y me dicen tía, a mí siempre me han dicho negro" (Profesional, Centro 6). “Es que en realidad no es tema, por ejemplo tú puedes decirle a alguien 'Juanito, el negrito', pero no hay una finalidad despectiva al decirle eso, o cuando le dices el peruano, o el ecuatoriano ese" (Profesional, Centro 6).

Lo anterior resignifica la discriminación estructural característica del imaginario cultural chileno (Tijoux, 2013a) y su naturalización en el contexto educativo. Por lo tanto, el "preámbulo en la autoconciencia del conflicto intercultural" ha devenido como una barrera en la prevención de la discriminación y ha sido considerado como una fase inicial en el camino de la vida democrática de la escuela intercultural, sobre todo por esta historia de "aspiración a la blancura" (Correa, 2016) que caracteriza la configuración de la identidad nacional. Entonces, si bien es una etapa peculiar para los contextos educacionales estudiados que responde a razones educativas e históricas, al mismo tiempo resulta una debilidad y expresa la necesidad de una estrategia encaminada al empoderamiento de las comunidades educativas respecto de la autoconciencia de sus propios preconceptos y el desarrollo de estrategias formativas.

\section{Discusión y conclusiones}

Los hallazgos en relación con las perspectivas de los profesionales sobre el conflicto en interculturalidad reflejan la complejidad y tensiones presentes en el desarrollo de la vida democrática de los contextos educativos estudiados. Sobre todo porque dichas visiones se expresan a lo largo de un continuum que abarca desde una tendencia a la ocultación y/o no reconocimiento del conflicto en interculturalidad e irrelevancia de la diferencia cultural en la fase cuantitativa, hasta la visibilidad del conflicto a partir de causas relacionadas - o no- con la singularidad cultural, el no reconocimiento del mismo y su enmascaramiento en la fase cualitativa.

Este carácter polifacético de las visiones de los profesionales se ha considerado una etapa inicial en la educación intercultural e inclusiva, como un "preámbulo de la autoconciencia del conflicto intercultural" para los centros estudiados. Se trata de una etapa propia ante el abrupto y tendiente aumento de la diversidad cultural en dichos establecimientos educacionales.

Si a esta condición se le adiciona la ausencia de un trabajo de sensibilización de la comunidad educativa respecto de sus propios preconceptos, ello deviene en obstáculo para el afianzamiento de la vida democrática en interculturalidad, ya que da lugar a barreras en la prevención de la discriminación y a un desaprovechamiento del conflicto para una cultura de la mediación y de comunicación intercultural. Como refiere Leiva (2012), el desarrollo de la 
comunicación intercultural hacia el interior de los centros es una vía para canalizar sus barreras para la cultura del diálogo y mediación en interculturalidad. Al respecto plantea que, entre sus principales características:

Integra competencias cognitivas y emotivas (para el establecimiento de relaciones positivas); permite la toma de conciencia de la diversidad; facilita la creación de un clima comunicativo cálido donde el diálogo es igualmente importante (Leiva, 2012, p. 66).

El desaprovechamiento del conflicto como espacio propicio para el logro de tales objetivos evidencia una visión transversal acerca del mismo como un problema, como un comportamiento que se ha demonizado hasta tal punto que es preciso minimizarlo, enmascararlo o negarlo. Por lo tanto, a ello también le subyace que en aquellos entornos educativos donde se reconoce la existencia de conflictos, existen malas prácticas.

Por otro lado, estos hallazgos guardan relación con las tipologías acerca del racismo identificadas por Riedemann y Stefoni (2015) en sus estudios con profesionales de establecimientos educacionales chilenos. $Y$ es que la negación de la discriminación, ya sea de manera directa, intencional o mitigada, subsume el no reconocimiento del conflicto y su enmascaramiento.

En definitiva, la educación intercultural ha planteado desafíos a las instituciones educativas chilenas con vistas a la prevención de la discriminación, para lo cual, como punto de partida, sus profesionales han de trabajar en comunidad a partir de la autoconciencia de sus propias teorías implícitas en torno al conflicto. Sobre todo porque:

Se ha de intentar, en definitiva, recuperar el tiempo para la reflexión y la investigación, así como huir del mero activismo sin fundamentación (...) de poco puede servir sugerir acciones, si estas no se aplican a la realidad propia (Murillo, 2008, p. 3).

Esto supone una actitud de valoración crítica y visión positiva de la interculturalidad en la escuela chilena del siglo XXI.

\section{Referencias}

Arnaiz, P. (2012). Escuelas eficaces e inclusivas: cómo favorecer su desarrollo. Educación Siglo XXI, 30(1), 25-44. Recuperado de http://revistas.um.es/educatio/article/view/149121/132111

Aguado, T. (2008). El enfoque intercultural en la formación del profesorado. Dilemas y propuestas. Revista Complutense de Educación, 19, 275-292. Recuperado de https://www.researchgate.net/publication/27594970_El_enfoque_intercultural_en_la_f ormacion_del_profesorado_Dilemas_y_propuestas

Barriga-Villanueva, R. (2008). Miradas a la interculturalidad. El caso de una escuela urbana con niños indígenas. Revista Mexicana de Investigación Educativa, 39, 1229-1254. Recuperado de http://www.redalyc.org/articulo.oa?id=1400390 
Bartolomé, M. (2002). Identidad y ciudadanía: un reto a la educación intercultural. Madrid: Narcea.

Castro, A. (2011). Estrategias de aculturación y adaptación psicológica y sociocultural de estudiantes extranjeros en la Argentina. Interdisciplinaria: Revista de Psicología y Ciencias Afines, 28(1), 115-130. Recuperado de www.redalyc.org/articulo.oa?id=18022327007

Cebrián, M. y Noguera, J. (2010). Conocimiento indígena sobre el medio ambiente y diseño de materiales educativos. Comunicar, 17(34), 114-125. Recuperado de https://www.revistacomunicar.com/verpdf.php?numero=34\&articulo=34-2010-14

Correa, J. (2016). La inmigración como "problema" o el resurgir de la raza. Racismo general, racismo cotidiano y su papel en la conformación de la Nación. En M. E. Tijoux (Ed.), Racismo en Chile. La piel como marca de la inmigración (pp. 35-49). Santiago de Chile: Editorial Universitaria.

Díaz, A. (2006). La educación en valores: avatares del currículum formal, oculto y los temas transversales. Revista Electrónica de Investigación Educativa, 8(1), 1-15. Recuperado de http://www.scielo.org.mx/pdf/redie/v8n1/v8n1a1.pdf

Dumrauf, A. y Menegaz, A. (2013). La construcción de un currículo intercultural a partir del diálogo de saberes: descripción y análisis de una experiencia de formación docente continua. Revista Electrónica de Enseñanza de las Ciencias, 12(1), 85-109. Recuperado de https://ri.conicet.gov.ar/handle/11336/23648

Essomba, M. (2006). Construir la escuela intercultural. Reflexiones y propuestas para trabajar la diversidad étnica y cultural. Barcelona: Graó.

Ibarrola-García, S. e Iriarte, R. (2013). La influencia positiva de la mediación escolar en la mejora de la calidad docente e institucional: percepciones del profesor mediador. Profesorado. Revista de Currículum y Formación del profesorado, 17(1), 367-384. Recuperado de http://www.ugr.es/ recfpro/rev171COL7.pdf

Jordán, J. y Castella, E. (2001). La educación intercultural, una respuesta a tiempo. Barcelona: Universitat Oberta de Catalunya, UOC.

Leiva, J. (2010). Educación intercultural y convivencia desde la perspectiva docente. Profesorado. Revista de Currículum y Formación del profesorado, 14(3), 251-274. Recuperado de https://www.researchgate.net/publication/50285771_educacion_intercultural_y_conviv encia_desde_la_perspectiva_docente

Leiva, J. (2012). Educación intercultural y convivencia. Málaga: Ediciones Aljibe.

Leiva, J. (2013). Relaciones interpersonales en contextos de educación intercultural: un estudio cualitativo. Revista de Educación y Humanidades, 4, 109-128. Recuperado de https://dialnet.unirioja.es/servlet/articulo?codigo $=4252334$

Leiva, J. (2015). Las esencias de la educación intercultural. Málaga: Ediciones Aljibe.

Leiva, J. (2016). Abriendo caminos de interculturalidad e inclusión en la escuela. Madrid: Dykinson, S.L. 
Leiva, J. (2017). La interculturalidad como respuesta para la prevención del fracaso escolar en contextos de riesgo. Anduli: Revista Andaluza de Ciencias Sociales, 16, 19-33. Recuperado de https://dialnet.unirioja.es/ejemplar/485063

Marín, M. y Venegas, M. (2009). Aspectos claves de la educación inclusiva. Salamanca: Inico. Recuperado de http://inico.usal.es/publicaciones/pdf/Educacion-Inclusiva.pdf

Mignolo, W. (2007). La idea de América Latina. Barcelona: Gedisa Editorial. Recuperado de http://www.ceapedi.com.ar/imagenes/biblioteca/libreria/420.pdf

Mignolo, W. (2008). La opción de-colonial: desprendimiento y apertura. Un manifiesto y un caso. Revista Tabula Rasa, 12, 243-281. Recuperado de http://www.revistatabularasa.org/numero-8/mignolo1.pdf

Murillo, P. (2008). ¿Ayuda u obstáculo? Sentido y credibilidad de la función asesora. Profesorado. Revista de Currículum y Formación del profesorado, 12(1), 1-10. Recuperado de https://idus.us.es/xmlui/handle/11441/63746

Riedemann, A. y Stefoni, C. (2015). Sobre el racismo, su negación, y las consecuencias para una educación antirracista en la enseñanza secundaria chilena. Polis, Revista Latinoamericana, 14(42), 191-216. Recuperado de https://scielo.conicyt.cl/pdf/polis/v14n42/art_10.pdf

Riedemann, A. (2008). La educación intercultural bilingüe en Chile: ‘ampliación de oportunidades para alumnos indígenas? Indiana, 25, 169-193. Recuperado de http://www.iai.spkberlin.de/fileadmin/dokumentenbibliothek/Indiana/Indiana_25/Indiana_25_169193_Riedemann.pdf

Soriano, E. (2004). La práctica educativa intercultural. Madrid: Ed. La Muralla.

Stefoni, C. (2010). Niños y niñas inmigrantes en Santiago de Chile. Entre la integración y la exclusión. En M. E. Tijoux (Ed.), Racismo en Chile. La piel como marca de la inmigración (pp. 89-103). Santiago de Chile: Editorial Universitaria.

Tijoux, M. E. (2016). Racismo en Chile. La piel como marca de la inmigración. Santiago de Chile: Editorial Universitaria.

Tijoux, M. E. (2013a). Las escuelas de la inmigración en la ciudad de Santiago: elementos para una educación contra el racismo. POLIS, Revista Latinoamericana, 12(35), 287-307. Recuperado de http://www.scielo.cl/scielo.php?script=sci_arttext\&pid=S0718-65682013000200013

Tijoux, M. E. (2013b). Niños marcados por la inmigración peruana: estigma, sufrimientos, resistencias. $\quad$ 2(61), 83-104. Recuperado de www.redalyc.org/pdf/105/10524674004.pdf

Villalta, M. (2016). Educación intercultural en Latinoamérica: análisis de las investigaciones de campo en la región. Revista Psicoperspectivas, 15(1), 118-131. Recuperado de www.redalyc.org/pdf/1710/171043532012.pdf 
Walsh, C. (2009). Interculturalidad y (de) colonialidad. Perspectivas críticas y políticas para el siglo $x x l, \quad 15(1), \quad 1-19 . \quad$ Recuperado de http://www.compitecuador.org/wpcontent/uploads/2017/09/Interculturaliad-y-decolonialidad.pdf 